\title{
Biological Applications of Platinum-Based Nanoclusters
}

\author{
Fanglue $\mathrm{Wu}^{*}$ \\ Department of Materials Science and Engineering, Texas A\&M University, USA
}

*Corresponding author: Fanglue Wu, Department of Materials Science and Engineering, Texas A\&M University, College Station, Texas 77843, USA.

To Cite This Article: Fanglue Wu, Biological Applications of Platinum-Based Nanoclusters. Am J Biomed Sci \& Res. 2020 - 6(6). AJBSR.MS.ID.001094. DOI: 10.34297/AJBSR.2020.06.001094.

Received: 䟧 December 02, 2019 ; Published: 泚 January 13, 2020

\begin{abstract}
This mini review briefly discusses the recent development of Pt-based nanoclusters towards biological applications. The ultra-small size plays a key role in the unique properties of Pt-based nanoclusters in these applications including biological imaging, enzyme-like property and cancer treatment. Future directions have also been shortly discussed.
\end{abstract}

Keywords: Platinum; Nanoclusters; Biological imaging; Enzyme; Cancer treatment

\section{Introduction}

Nanomaterials are materials which exist on a nanometer scale in at least one dimension. These materials, especially noble metal nanoparticles, exhibit distinct physical and chemical properties compared to their bulk counterparts due to the high surface to volume ratio and the quantum confinement effect, which make them highly compatible in materials science and biological applications. When the sizes are less than $2 \mathrm{~nm}$, nanoparticles become nanoclusters, whose electronic structures change from a continuous band into a discrete molecular-like orbital levels. Such unique electronic properties combined with the good biocompatibility and photostability, suggesting promising potentials of these noble metal nanoclusters for biological applications [1]. This mini review will focus on Platinum (Pt) nanoclusters and the corresponding biological applications specifically in biological imaging, enzymelike properties and cancer treatment.

\section{Discussion}

\section{Biological Imaging}

Biological imaging provides unique advantages in cancer identification and drug delivery [2]. One of the most critical factors for successful biological imaging is the use of stable, biocompatible and sensitive markers [3]. Traditional markers including organic dyes and fluorescent proteins often experience stability concerns for long-term experiments. Quantum dots markers have disadvantages such as biocompatibility issues for in vivo use. In contrast, Pt nanoclusters illustrate high sensitivity in long-term experiments and biocompatibility, making them highly suitable for biological imaging. For example, it has been reported that Pt nanoclusters attached by polyamine could be used for staining in hematopoietic system [4]. In addition, cell membranes have been imaged by blue mercaptoacetic acid protected Pt nanoclusters, where the antibody receptors were expressed [5].

\section{Enzyme-Like Activities}

Except for the excellent photoluminescence properties for bioimaging, protein capped Pt nanoclusters have also illustrated enzyme-like properties, i.e. peroxidase, oxidase and catalase [6]. Peroxidases are type of enzymes that reduce the lipid peroxide or hydrogen peroxide, and high peroxidase activities of Pt-based nanoclusters have been reported by Wei and coworker [7]. Based on the inhibition behavior of the peroxidase enzymatic activities between $\mathrm{Pt}$ and $\mathrm{Hg}$, Pt nanoclusters have been proposed and utilized for the detection of toxic metal ions [8]. Oxidases are type of enzymes that promote oxidation by molecular Oxygen $\left(\mathrm{O}_{2}\right)$.

Tseng and coworkers have shown that lysozyme ligand protected Pt nanoclusters could catalyze the oxidation reactions of organic substances such as dopamine and the degradation mechanism of organic pollutants by $\mathrm{Pt}$ nanoclusters have also been proposed [9]. Catalases are type of enzymes that decompose hydrogen peroxide into $\mathrm{O}_{2}$ and $\mathrm{H}_{2} \mathrm{O}$. Nie, et al. have reported that the 
Platinum-ferritin nanoclusters could catalyze the decomposition of $\mathrm{H}_{2} \mathrm{O}_{2}$, which further reduce the 5-Diethoxyphosphoryl-5-methyl1-pyroline-N-oxide (DEPMPO)/OH' adduct signal in a $\mathrm{H}_{2} \mathrm{O}_{2} / \mathrm{UV}$ DEPMPO spin trap system [10].

\section{Cancer Treatment}

Platinum-Based drugs are widely used compounds for treatments of head, neck, cervical and lung cancers [11]. DNAPt adducts produced by cisplatin and other analogues are wellknown for their anti-tumor activities decades ago. However, these drugs demonstrate little effect on breast, liver, and prostate cancers, as well as similar tumor sensitivity and susceptibility to tumor resistance. To overcome this, demethylcantharidin has been employed to introduce the selectivity of anti-tumor behavior towards liver cancer cells [12]. Additionally, demethylcantharidinplatinum complexes have also shown to be free from cross resistance with cisplatin. Chien et al. have reported a dendrimercapped Pt nanocluster for targeting breast cancer cells [13]. Xia et al. have demonstrated polypeptide protected Pt nanoclusters could accelerate the release of Pt ions and overcome the cisplatin resistance problems [14].

\section{Conclusion}

Due to ultra-small size, Pt nanoclusters have illustrated distinct electronic properties compared to the bulk materials. Combined with the good biocompatibility and photoluminescence, $\mathrm{Pt}$ nanoclusters have demonstrated exciting potential for biological applications such as, biological imaging, enzyme-like property and cancer treatment. Future directions include synthesizing Pt nanoclusters with improved florescence character, enhancing enzyme activities and preparation of new ligand groups for targeting tumor cells with lower resistivity.

\section{References}

1. Shang L, Dong S, Nienhaus GU (2011) Ultra-small fluorescent metal nanoclusters: synthesis and biological applications. Nano today 6(4): 401-418.

2. Gong X, Zhang Q, Gao Y, Shuang S, Choi MM, et al. (2016) Phosphorus and nitrogen dual-doped hollow carbon dot as a nanocarrier for doxorubicin delivery and biological imaging. ACS applied materials \& interfaces 8(18): 11288-11297.

3. Fernández-Suárez M, Ting AY (2008) Fluorescent probes for superresolution imaging in living cells. Nature reviews Molecular cell biology 9: 929-943.

4. Kieler-Ferguson, HM, Fréchet JM, Szoka FC (2013) Clinical developments of chemotherapeutic nanomedicines: polymers and liposomes for delivery of camptothecins and platinum (II) drugs. Wiley Interdisciplinary Reviews: Nanomedicine and Nanobiotechnology 5(2): 130-138.

5. Tanaka SI, Miyazaki J, Tiwari DK, Jin T, Inouye Y (2011) Fluorescent platinum nanoclusters: synthesis, purification, characterization, and application to bioimaging. Angewandte Chemie International Edition 50(2): 431-435.

6. Meng X, Zare I, Yan X, Fan K (2019) Protein-protected metal nanoclusters: An emerging ultra-small nanozyme. Wiley Interdisciplinary Reviews: Nanomedicine and Nanobiotechnology.

7. Li W, Chen B, Zhang H, Sun Y, Wang J, et al. (2015) BSA-stabilized Pt nanozyme for peroxidase mimetics and its application on colorimetric detection of mercury (II) ions. Biosensors and Bioelectronics 66: 251258.

8. Fu Y, Zhao X, Zhang J, Li W (2014) DNA-based platinum nanozymes for peroxidase mimetics. The Journal of Physical Chemistry C 118(31): 18116-18125

9. Yu CJ, Chen TH, Jiang JY, Tseng WL (2014) Lysozyme-directed synthesis of platinum nanoclusters as a mimic oxidase. Nanoscale 6(16): 96189624.

10. Fan J, Yin JJ, Ning B, Wu X, Hu Y, et al. (2011) Direct evidence for catalase and peroxidase activities of ferritin-platinum nanoparticles. Biomaterials 32(6): 1611-1618.

11. Rosenberg B (1999) Platinum complexes for the treatment of cancer: Why the search goes on. Cisplatin. Chemistry and biochemistry of a leading anticancer drug pp. 3-27.

12. Ho YP, Au-Yeung SC, To KK (2003) Platinum-based anticancer agents: Innovative design strategies and biological perspectives. Medicinal research reviews 23(5): 633-655.

13. Chien CT, Yan JY, Chiu WC, Wu TH, Liu CY, et al. (2013) Caged Pt Nanoclusters Exhibiting Corrodibility to Exert Tumor-Inside Activation for Anticancer Chemotherapeutics. Advanced Materials 25(36): 50675073.

14. Xia H, Li F, Hu X, Park W, Wang S, et al. (2016) pH-sensitive Pt Nanocluster assembly overcomes Cisplatin resistance and heterogeneous Stemness of hepatocellular carcinoma. ACS central science 2(11): 802-811. 